\title{
Marx et Engels en Allemagne. À propos de quelques publications récentes
}

Jean-Numa Ducange

\section{CpenEdition}

Journals

Édition électronique

URL : http://journals.openedition.org/ifha/493

DOI : 10.4000/ifha.493

ISSN : 2198-8943

\section{Éditeur}

IFRA - Institut franco-allemand (sciences historiques et sociales)

\section{Édition imprimée}

Date de publication : 30 septembre 2012

Pagination : 290-307

ISSN : 2190-0078

\section{Référence électronique}

Jean-Numa Ducange, "Marx et Engels en Allemagne. À propos de quelques publications récentes »,

Revue de l'IFHA [En ligne], 4 | 2012, mis en ligne le 14 février 2013, consulté le 19 avril 2019. URL

http://journals.openedition.org/ifha/493 ; DOI : 10.4000/ifha.493

Ce document a été généré automatiquement le 19 avril 2019.

(CIFHA 


\title{
Marx et Engels en Allemagne. À propos de quelques publications récentes
}

\author{
Jean-Numa Ducange
}

1 Pourquoi tenter d'établir un panorama des publications de et sur Marx et Engels en Allemagne plus de vingt ans après la chute du mur de Berlin? En premier lieu le relatif regain d'intérêt pour leur oeuvre et sa réception sur le long terme à travers plusieurs ouvrages mérite en soi une certaine attention. Surtout, dans un pays où la référence à Marx et au marxisme a été surdéterminée pendant quarante ans par la partition de 1949 et l'existence de la RDA, il n'est pas anodin de relever qu'aujourd'hui nombre d'Allemands qui souhaitent s'engager (ou qui se situent dans cette perspective à court terme) dans un travail de recherche en histoire, économie, philosophie ou toute autre science sociale sont nés au moment de l'effondrement du « socialisme réel », qu'ils ne peuvent connaître qu'à travers des travaux historiques ou la mémoire transmise par les habitants de l'ancienne Allemagne orientale. Leur rapport au "marxisme» est nécessairement très différent de ceux qui ont évolué du temps des deux Allemagnes. C'est donc dans un tout autre climat intellectuel qu'il faut appréhender les nouvelles parutions sur Marx, en ayant à l'esprit les forts héritages de la période d'avant 1989, que de multiples débats, liés à l'abondance exceptionnelle des publications sur la RDA, soulignent. Avec la crise économique intervenue en 2007-2008, les médias allemands ont souligné à plusieurs reprises un certain regain d'intérêt pour Marx, qui s'est traduit par des ventes importantes du livre I du Capital. Au niveau politique, l'organisation de jeunesse de Die Linke $^{1}$ a lancé en 2008-2009 une campagne pour constituer des groupes de lectures du Capital qui ont eu, à leur niveau, un certain succès. Pour la première fois depuis la réunification, l'Allemagne a donc connu un certain frémissement autour de Marx, alors même que son nom était encore il y a peu presque systématiquement associé au régime du SED.

2 Nous présenterons ici une série de publications, nullement exhaustive, parues ces dernières années et dont le contenu nous paraît représentatif des approches actuelles. En 
premier lieu on évoquera l'édition scientifique de Karl Marx et Friedrich Engels, puis un ouvrage introductif à l'œuvre de Marx adressé à un large public, avant d'étudier plusieurs contributions traitant des lectures, usages et réceptions des marxismes en Allemagne et au-delà. Autant de publications qui permettent d'ouvrir une réflexion méthodologique sur l'histoire complexe des marxismes.

\section{La poursuite d'une entreprise : l'édition de la MEGA}

En tant que telle l'histoire des éditions de Marx pourrait être l'objet d'un ouvrage prenant en compte la dimension internationale, intellectuelle et politique du phénomène ${ }^{2}$. Elle permettrait probablement de mieux appréhender une des références centrales du vingtième siècle qui a parcouru le monde entier. Nous reviendrons plus loin sur la façon dont on peut envisager l'histoire de cette réception; pour le moment signalons que, en 2012, la totalité des textes et manuscrits de Marx et Engels n'a pas encore été publiée dans le cadre du très ambitieux projet de la Marx Engels Gesamtausgabe : formellement, la MEGA n'a pour le moment publié que la moitié des volumes prévus (120 au total) même si désormais, à quelques exceptions, on peut considérer que l'essentiel du corpus est connu des spécialistes ${ }^{3}$.

Le projet de la MEGA, qui vise à établir la totalité des textes de Marx et Engels en quatre sections (I œuvres, articles et esquisses; II manuscrits et textes préparatoires du Capital ${ }^{4}$, III correspondances ; IV cahiers de notes et notes en marge des ouvrages consultés et lus) a été lancé conjointement en 1975 par les Instituts du marxisme-léninisme de Berlin-Est de Moscou, reprenant le premier projet tragiquement interrompu dans les années 1930 en URSS de David Riazanov. Auparavant en RDA dès 1956 avait été lancée l'édition des Marx Engels Werke regroupant la majeure partie des textes (45 volumes au total dont la plupart édités dans les années 1960-1970) mais sans perspective d'exhaustivité, tandis que l'entreprise était très marquée par la politique du régime est-allemand ${ }^{5}$. La MEGA visait quant à elle un objectif davantage scientifique, bien que la tutelle politique soit restée importante jusqu'en 1989. Pour cette raison, elle a d'ailleurs failli subir le même sort après 1990 que nombre de projets menés par ces instituts liés organiquement aux partisÉtats, à savoir la disparition pure et simple. Mais l'Institut international d'histoire sociale d'Amsterdam (qui possède la plus grande partie des manuscrits originaux de Marx et Engels provenant des archives du SPD récupérés par cet institut après 1933) a, par l'intermédiaire d'une fondation, l'IMES (Internationale Marx-Engels Stiftung), réussi à sauver la MEGA de la disparition. Désormais dénué de l'arrière-plan idéologique d'avant 1989 qui était particulièrement présent dans l'« Einleitung " marxiste-léniniste de chaque volume, le projet a pu être poursuivi, grâce notamment à l'ouverture en direction de nouveaux chercheurs, tout en assurant partiellement le maintien des équipes antérieures. Publiés par l'Akademie Verlag (et non plus par Dietz Verlag, maison historique du mouvement ouvrier allemand), les volumes sont supervisés par une équipe de chercheurs internationaux, au sein de laquelle on peut observer une place importante des collègues du continent asiatique ${ }^{6}$. Ainsi, la question controversée de l'établissement des manuscrits de l'Idéologie allemande dont la première publication remonte aux années 1930, a fait l'objet récemment d'un débat international mobilisant des universitaires sud-coréens et japonais, aboutissant par exemple à l'eventualité de l'attribution de la paternité de certains manuscrits à Joseph Weydemeyer et non plus uniquement à Marx et Engels ${ }^{7}$. 

référence pour des chercheurs d'horizons divers, au-delà de la seule marxologie. Les deux dernières livraisons de la MEGA, qui contiennent les textes d'Engels après la mort de Marx en 1883, montrent l'ampleur du travail d'érudition mené par les chercheurs du projet $^{9}$ : pour le volume I/32 à 528 pages de textes d'Engels correspondent pas moins de 1 590 pages d'appareil critique incluant une contextualisation très précise de chaque texte et ses variantes éventuelles selon les différents manuscrits.

8 L'intérêt des textes publiés ici est multiple ; nous soulignerons avant tout leur singularité au regard de ce qu'ils peuvent apporter au regard de la connaissance antérieure de l'œuvre de Friedrich Engels et de sa réception historique. Disposer de l'intégralité des textes d'Engels après 1883 permet de reprendre, dans un contexte moins marqué par les affrontements idéologiques, la question de l'invention du « marxisme » par Engels et ses épigones comme Karl Kautsky, marxisme souvent considéré comme précurseur d'un matéralisme fossilisé, antidialectique et contraire à l'esprit de Marx, qui aurait été développé par la Deuxième Internationale puis repris d'une certaine manière par l'Internationale communiste stalinisée. Incarnée par Maximilien Rubel et toute une tradition d'interprétation ${ }^{10}$, que l'on peut rapprocher des analyses de Karl Korch sur le marxisme, cette tradition a longtemps reçu un accueil important dans la marxologie occidentale, leur critique paraissait alors en adéquation la dénonciation du «marxismeléninisme » professé dans les pays $\mathrm{du}$ « socialisme réel ».

Plus de vingt ans après la dislocation de l'URSS, il convient de prendre ses distances par rapport à ces analyses afin de reconstruire historiquement les multiples réalités successives des marxismes. Le travail d'Engels a consisté avant tout à faire connaître ses textes et ceux de Marx auprès des mouvements ouvriers organisés alors en pleine croissance. Sont ici réunies pour la première fois à notre connaissance l'intégralité des préfaces rédigées par Engels dans cet objectif, toujours ici - suivant en cela la logique de la MEGA, différente des anciennes Marx Engels Werke qui avaient toujours publié les textes en allemand - retranscrites dans leur langue originale. Engels justifie chacune de ces introductions, n'hésitant pas à souligner les insuffisances de tel ou tel texte selon la date et le(s) pays concerné(s). L'ensemble permet aux historiens, philosophes et à tous ceux qui s'intéressent à cette oeuvre d'envisager une étude précise des transferts d'un pays à l'autre et une « histoire croisée ${ }^{11}$ » des trajectoires des textes à l'échelle internationale. L' Apparat contient à cet égard des éléments de contextualisation précieux sur les conditions d'introduction de chacun de ces textes, y compris les plus courts.

Un autre grand atout de cette édition vient de la publication intégrale des traductions auxquelles Engels a collaboré. Le parti pris ici est donc d'offrir au-delà des « seuls » textes 
rédigés par Engels lui-même les traductions révisées et relues par lui. Le lecteur dispose ainsi de la première traduction de Laura Lafargue du Manifeste du parti communiste, entre autres. Dans le même esprit sont publiés des textes de dirigeants socialistes qui doivent quelque chose à la relecture d'Engels (Paul Lafargue, Karl Kautsky...). Ajoutons enfin les interviews d'Engels avec différents journaux européens, de l'Italie à la Russie, qui confèrent à l'ensemble une cohérence fournissant de nombreux matériaux peu connus, du moins souvent peu cités dans les travaux consacrés à ce sujet.

11 Ainsi réunis, ces textes permettront probablement le développement de nouvelles approches, moins centrées sur les quelques "grands textes" comme la Critique du programme d'Erfurt au contenu d'une importance capitale, mais dont la publication est intervenue pour la première fois après la mort d'Engels en 1901, contrairement à d'autres textes moins connus, présentés ici dans ces volumes. Ils permettent de s'émanciper des héritages "marxistes-léninistes" les plus caricaturaux qui avaient figé dans le marbre quelques textes de "Marx-Engels-Lénine » pour redécouvrir la cohérence propre des écrits d'Engels en leur temps, et également de critiquer de facto les approches très idéologiques qui réduisent Engels à un rôle de « fossoyeur » de Marx. En effet, s'il existe des différences véritables entre Marx et Engels au niveau théorique, l'effort du second pour publier le premier - sans même revenir sur les textes écrits ensemble - est tel qu'il rend historiquement nécessaire de rapprocher les deux pour comprendre les héritages réciproques, et ce sans anathème particulier. Les efforts spécifiques pour poursuivre la publication du Capital - que le lecteur érudit pourra lire en miroir avec les sections II et IV de la MEGA - et d'autres textes plus directement politiques, le montrent amplement. L'Apparat fournit par ailleurs, en plus de la contextualisation, un riche bilan historiographique pour les textes qui ont eu une longue postérité dans les débats théoriques, à l'image du dernier texte d'Engels de 1895, l'introduction aux Luttes de classes en France en 1848-1850 qui avance pour la première fois l'idée d'un passage non violent au socialisme par la médiation du suffrage universel. Considéré ultérieurement comme la «bible» des révisionnistes proches d'Eduard Bernstein qui souhaitaient autour de 1900 dégager la social-démocratie du "révolutionnarisme » verbal issu du Manifeste du parti communiste, le texte connut des usages multiples avant que la version originale du texte, non amputée comme l'avait été la version initiale de 1895, ne soit publiée en URSS au début des années 1930, provoquant à nouveau un débat à l'échelle internationale entre sociaux-démocrates et communistes. Enfin, au regard de travaux récents sur Engels et le mouvement ouvrier européen de son époque, on mesure combien ces textes seront utiles aux historiens ; l'exemple de la biographie de Tristram Hunt ${ }^{12}$, fort érudit sur le rapport d'Engels à l'Angleterre, mais lacunaire sur les autres pays pour la dernière période, montre que le « vieil Engels » est encore digne d'intérêt.

13 En parallèle de la publication des volumes de la MEGA paraît chaque année un Jahrbuch regroupant des contributions érudites sur Marx et Engels de nature très différente (historique, philosophique, économique, etc.) ou philologique (le Jahrbuch de 2003 ayant par exemple publié une partie des manuscrits inédits de l'Idéologie allemande), ensemble accompagné de comptes rendus souvent denses sur diverses publications relatives au même thème. Le Jahrbuch dresse également un panorama régulier de l'avancée des éditions scientifiques de Marx et Engels dans d'autres langues. Les deux dernières livraisons de 2009 et 2010 donnent la mesure de la richesse de l'ensemble ${ }^{13}$. Dans l'édition de 2009 sont regroupées les contributions d'une journée d'étude consacrée à la place de l'individu chez Marx et l'utilité de sa pensée pour saisir la réalité de la sphère 
intellectuelle en régime capitaliste. D'autres textes s'inscrivent plus directement dans la «marxologie »: l'un est ainsi consacré au regard du jeune Engels au début des années 1840 sur les entreprises communautaires socialistes et les sources utilisées qui lui ont permis de mieux les connaître, ouvrant des perspectives sur ses méthodes de travail et ses premiers développements politiques. Le Jahrbuch de 2010 contient quant à lui notamment une série d'articles du plus grand intérêt sur le rapport de Marx et Engels aux nationalismes de leur temps, que l'on doit lire en miroir d'un long compte rendu publié dans le même volume consacré à l'ouvrage de Kevin B. Anderson, Marx at the Margins: On Nationalism, Ethnicity, and Non-Western Societies paru aux États-Unis en $2010^{14}$. Sur ces questions, le regard porté sur les écrits de Marx (distinct d'Engels de ce point de vue) a considérablement changé grâce à des chercheurs connaissant l'intégralité du corpus et ayant opéré une stricte contextualisation des textes. À un Marx russophobe dans les années 1840-1850 a été progressivement opposé un Marx des années 1870-1880, plus sensible aux spécificités "asiatiques » et aux modalités possibles de développement du socialisme dans différents pays. L'exemple le plus célèbre est la façon dont Marx considère la communauté rurale (mir) en Russie, dont les traditions égalitaires peuvent avoir un effet positif. Certains textes, comme un brouillon de lettre à la militante russe Véra Zassoulitch étaient connus depuis longtemps ${ }^{15}$. Néanmoins l'article de Skadi Krause a l'immense mérite ici de proposer un regard global critiquant les visions les plus courantes, très répandues dans une partie du monde académique, d'un Marx européocentré incapable de penser des transitions politiques et sociales hors du schéma capitaliste occidental ${ }^{16}$. Dans un autre article suggestif, Lucia Pradella montre les changements opérés par Marx entre l'édition allemande du Capital de 1867 et l'édition française de 1875 qu'il a lui-même entièrement relue ${ }^{17}$; à partir d'une étude érudite sur ses notes, l'auteure montre que Marx porte une attention plus grande à la question coloniale et aux sociétés encore peu touchées par le développement du capitalisme dans l'édition de 1875. Dans le même esprit, le compte rendu détaillant le contenu de l'ouvrage de Kevin B. Anderson ${ }^{18}$ montre que ces problématiques sont au cœur des recherches actuelles, Anderson fondant sa réflexion sur la question nationale chez Marx à partir de textes méconnus ou rarement explorés sous cet angle. Contre les lectures " orientalistes » proposées par Edward Said, il souligne que Marx a accordé, après une période très " européenne » autour des années 1848, une importance croissante aux développements spécifiques de l'Inde, dont il a pris tôt conscience de l'importance. Ce qu'il y a de plus marquant dans l'étude d'Anderson par rapport à d'autres travaux est l'usage que l'auteur fait de textes habituellement peu mobilisés : cahiers de notes, ouvrages annotés, articles de journaux... D'importants développements sont à souligner sur les Grundrisse (manuscrits préparatoires du Capital) qui montrent d'après lui que la pensée marxienne est fondamentalement non linéaire et ouverte sur des problématiques plus complexes que la seule logique "capitalisme - révolution - socialisme », insistant sur l'importance de la prise en compte des sociétés non occidentales avec le concept de «mode de production asiatique ». Le propos du chercheur américain, qui ne manquera pas de susciter de nouveaux débats, informe par ailleurs sur l'utilité des travaux à venir publiés par la MEGA : le dernier grand projet de Marx consistait à écrire une Histoire universelle, en quelque sorte l'équivalent historique du Capital mais remontant aux origines de l'homme, dont il nous reste des fragments et notes éparses et que le projet d'édition envisage de publier intégralement dans les prochaines années. brouillons et esquisses en les confrontant aux œuvres les plus célèbres, sans oublier les 
textes d'apparence plus secondaire publiés à l'époque (brefs articles dans des journaux, entretiens, etc.), voilà assurément un des grands axes de la recherche menée par les équipes de la MEGA qui autorise des développements érudits sur l'élaboration et la diffusion de certains textes, mais également des réflexions sur les théories marxiennes, à l'image du renouvellement en cours autour des questions du développement des sociétés non occidentales.

\section{Introduire Marx aujourd'hui}

En parallèle à l'édition des textes, une longue tradition d'abrégés, bréviaires et autres textes introductifs à Marx a toujours existé, tant il allait de soi que seul un propos synthétique pouvait permettre de comprendre la pensée marxienne ou marxiste, souvent complexe, voire incompréhensible, pour qui ne disposait pas d'un solide bagage théorique et historique. Cette tradition de publication "populaire » visant à rendre intelligible par exemple les grands mécanismes du capitalisme tels qu'ils sont exposés dans le Capital, mérite donc une attention spécifique. La tradition était d'abord militante et engagée : Marx lui-même a publié des courtes brochures comme Salaires, prix et profits ${ }^{19}$, puis les sociaux-démocrates allemands dans les années 1880-1890, repris en cela par des socialistes de toute l'Europe, ont cherché à diffuser massivement ce type d'ouvrages. L'un des plus célèbres pour les pays germaniques, traduit en de très nombreuses langues, fut le résumé du Capital de Karl Kautsky, Karl Marx' ökonomische Lehren ${ }^{20}$, vertement critiqué ensuite pour ses aspects très " économistes » et mécanistes, mais dont le succès a permis un accès "populaire » à certains concepts marxistes. Beaucoup plus tard, à partir des années 1960-1970, cette tradition s'est ensuite enrichie d'un nouveau type de publication, contemporain de l'extension des sciences sociales et du succès planétaire du marxisme, à savoir d'ouvrages introductifs à Marx publiés par des maisons d'éditions non militantes et souvent universitaires, certains ouvrages se situant à la charnière des deux sphères, académique et politique. L'histoire des publications directement politiques peut être ainsi reliée à l'abondante production de manuels universitaires qui ont pu servir les unes comme les autres alternativement d'introduction à Marx auprès d'un large public. Après le déclin de ces deux types de publications dans les années 1980, et bien qu'elles se soient maintenues jusqu'à nos jours, un nouveau type d'ouvrage semble apparaître depuis une quinzaine d'années, empruntant des traits aux deux précédents mais avec une originalité spécifique : "retour à Marx » et, pourrait-on dire, à Marx seul. L'ouvrage paru en 2007 Karl Marx zur Einführung de Rolf Peter Sieferle aux éditions Junius en est un bon exemple ${ }^{21}$. Cinq parties sont nettement définies: «le programme du socialisme scientifique » où l'auteur présente le rapport Hegel - Marx et l'élaboration de la conception matérialiste de l'histoire ; «l'anatomie de la société bourgeoise » qui traite des principales conceptions économiques de Marx; «les tendances du capitalisme » où est discutée la façon dont Marx envisage notamment le dépassement du capitalisme. Les deux dernières parties "Révolution et socialisme » et "Marx et le marxisme » abordent la place du politique chez Marx et les appropriations postérieures de sa pensée. Clair et précis, l'ouvrage constitue une bonne introduction à la pensée marxienne, fondée sur une lecture attentive des textes. Une des difficultés majeures de ce type d'exercice tient justement à la lecture de ces derniers, dont l'établissement n'a été que très progressif, rendant par-là même toute lecture in extenso extrêmement complexe et susceptible de provoquer de multiples controverses. Sieferle a de ce point de vue raison de relever en introduction à sa partie 
sur l'histoire du marxisme le décalage entre les textes publiés du vivant de Marx et ceux édités ultérieurement pendant plusieurs décennies ${ }^{22}$. De ce fait, toute connaissance de Marx a longtemps été surdéterminée par la publication de manuscrits fondamentaux qui ont modifié durablement les lectures de Marx, souvent contre le " marxisme-léninisme " officiel en vigueur dans les pays du bloc soviétique. L'ouvrage s'achève par l'invention du «marxisme " par Engels ${ }^{23}$, la distance philosophique qui sépare ce dernier de Marx; un aperçu sur le rapport entre les mouvements politiques et le marxisme, bref mais stimulant, clôt l'ouvrage. Enfin, caractéristique en cela des appréciations les plus courantes, l'auteur propose un retour à Marx en rupture avec ses appropriations politiques traditionnelles, pointant une œuvre « hétérogène et expérimentale ${ }^{24} »$. Un tel constat pose directement problème des interprétations des textes de Marx, en particulier au vingtième siècle, question qui a fait l'objet d'importants travaux parus ces dernières années.

\section{L'histoire des lectures de Marx et des marxismes}

16 Jan Hoff propose dans un ouvrage ambitieux, Marx Global $^{25}$, d'analyser les discussions théoriques autour du Capital de Marx à l'échelle mondiale à partir d'une bibliographie abondante, présente en fin de volume et outil d'une grande utilité pour disposer d'une géographie de la réception de l'œuvre économique de Marx. Moins qu'une histoire éditoriale, il s'agit davantage d'une histoire des débats autour de la critique de l'économie politique telle qu'elle est envisagée par Marx. Ordonné selon quelques grands thèmes, avec au sein de chaque section une dimension géographique importante (par pays ou continent), l'ouvrage de Jan Hoff constitue une admirable synthèse qui fera date, mobilisant une impressionnante connaissance de la bibliographie à l'échelle de plusieurs décennies, tout en montrant un regard informé sur les débats actuels autour de l'œuvre marxienne. Laissant volontairement de côté les approches marxistes historiques ou plus directement politiques, Hoff étudie en revanche toutes les lectures de Marx et des marxismes ayant eu comme prétention d'élaborer une théorie globale du capitalisme dans la lignée du Capital. Le point de vue de l'auteur se situe résolument dans une tradition althussérienne, selon laquelle la critique de l'économie politique est le cœur du propos marxien. Notons cependant que la première partie de l'ouvrage peut servir d'introduction historiographique plus globale, puisqu'elle cite toutes les histoires importantes du marxisme parues jusqu'à la publication de l'ouvrage. Tout en s'inscrivant dans la continuité des travaux antérieurs, Hoff tente ainsi une première "world history " des discussions autour de Marx pour se démarquer des approches trop centrées sur l'Europe. Ce n'est pas le moindre de ses mérites : le lecteur prend ainsi connaissance de noms peu familiers de marxologues et marxistes japonais, notamment de l'école d'Uno ${ }^{26}$, fournissant des clefs de lectures pour le présent, marqué par un fort intérêt marxologique au Japon que l'on retrouve dans l'équipe chargée de l'établissement des textes de la Marx Engels Gesamtausgabe ${ }^{27}$; de même pour ce qui concerne la Chine où émergent des débats entre 1965 et 1985 dans le double contexte de la première édition critique et des mutations économiques du " socialisme de marché28 ". L'auteur n'oublie pas les débats au sein du bloc soviétique, où il relève par exemple en RDA une école féconde à distance de la doxa officielle ${ }^{29}$. L'histoire de ces controverses est mise en relation avec l'édition des manuscrits, depuis les premières publications en URSS dans les années 1930 de l'Idéologie allemande, des Manuscrits de 1844 et des Grundrisse. La chronologie de la réception 
ultérieure des textes sert de trame générale à son propos : fort regain d'intérêt dans les années 1960-1970, puis déclin à partir des années 1980. Un des intérêts du traitement mondial de l'objet permet d'affiner cette courbe ascendante et descendante bien connue, valide en réalité pour l'Europe occidentale mais différente ailleurs, à l'image du continent asiatique et dans une moindre mesure latino-américain, où le rapport à Marx paraît beaucoup plus dynamique que dans un pays comme la France, malgré là aussi un certain « retour » récent. Les cents dernières pages de l'ouvrage sont consacrées aux tendances actuelles de la discussion, en particulier à la très grande importance du concept de fétichisme comme clef d'interprétation du monde social, témoignant du succès des thématiques de l'école de Francfort. La validité de la théorie de la valeur telle qu'elle est définie par Marx dans le Capital a fait l'objet aussi d'importants débats, prolongeant une longue tradition, la remise en cause de la théorie de la valeur étant en effet débattue au moins depuis le débat révisionniste en 1899... Citons enfin, sans exclusive, le développement du marxisme analytique farouchement anti-hégélien et se situant dans une perspective critique des interprétations orthodoxes de Marx, ou encore le problème de la notion de crise dans le Capital. Les débats les plus contemporains ont été réactivés par l'édition presque arrivée à son terme des manuscrits du Capital de Marx dans la deuxième section de la MEGA, permettant de disposer des intuitions et travaux de départ de Marx alors que les choix d'Engels de publier certains manuscrits sous la forme des livres II et III, qui avaient longtemps fait autorité, sont désormais remis en cause.

L'ouvrage d'Ingo Elbe Marx im Westen publié au même moment se situe dans une démarche similaire ${ }^{30}$. D'un format plus important et beaucoup plus limité en terme géographique - l'auteur se concentre sur la RFA - l'ouvrage présente dans le détail les diverses lectures de Marx depuis les années 1960, avec un souci d'exhaustivité qui en fait une contribution de tout premier plan. Il établit notamment la nette filiation, quelque peu oubliée aujourd'hui au regard de son évolution ultérieure, entre Marx et l'école de Francfort. Ces lectures ouest-allemandes strictement contextualisées montrent qu'elles furent dépendantes d'introduction de marxistes étrangers comme Poulantzas ou Althusser. Divisé en trois grands ensembles (théorie de la valeur, conception de l'État et théorie de la révolution), le propos permet entre autres de mieux saisir la généalogie d'auteurs influents comme Wolfang Fritz Haug et Michael Heinrich, responsables respectifs des deux revues marxistes les plus importantes encore aujourd'hui, Das Argument et Prokla. Une telle étude est à rapprocher de l'une des entreprises «marxologiques» les plus importantes d'Allemagne, à savoir l'Historisch-kritische Wörterbuch des Marxismus publié depuis 1994 sous la direction d'un collectif de chercheurs animant Das Argument et largement ouvert sur le monde comme au niveau méthodologique.

Quoique très informés, les ouvrages de Jan Hoff et Ingo Elbe reconduisent un certain nombre d'impasses propres à ce type d'histoire, que l'on pourra qualifier d'intellectuelle ou conceptuelle ${ }^{31}$. La façon dont Jan Hoff a présenté récemment un panorama des publications de et sur Marx en Allemagne dans une revue américaine paraît à cet égard révélatrice de la méthode proposée ${ }^{32}$ : bien que le contexte politique soit évoqué au début de l'article, l'ensemble reste essentiellement confiné à la production d'auteurs universitaires, et on ne repère que très marginalement des interrogations sur le poids de la référence à Marx et aux marxismes dans les organisations de gauche, syndicales et politiques ou encore de manière plus générale dans les sciences sociales. La production historique d'inspiration marxiste ou marxienne n'a pas droit de cité, y compris celle 
ayant mobilisé les aspects économiques de l'œuvre de Marx pour interroger l'histoire, et un problème majeur comme le lien entre l'histoire sociale et le marxisme est à peine effleuré. Il n'est certes guère possible de traiter dans un ouvrage et a fortiori dans un article de toutes ces influences mais se limiter stricto sensu aux lectures liées à la critique de l'économie politique nous semble aboutir méthodologiquement à des impasses importantes, tout simplement en décalage avec la diversité des textes publiés par Marx et Engels de leur vivant. En effet, on l'a déjà souligné, l'histoire des bréviaires et des introductions fait pleinement partie de l'histoire du marxisme depuis le vivant de Marx; par définition, la lecture de ce type de littérature répétitive peut être rebutant et paraître sans intérêt, mais c'est probablement à travers leurs lectures et leur stricte contextualisation que l'on peut mesurer ce qu'a été la diffusion d'un certain marxisme. De ce point de vue, les approches d'Ingo Elbe et Jan Hoff contre l'« engelsianisme », si elles ont leur légitimité théorique, nous semblent peu à même de comprendre le lien qui s'est tôt établi entre la pensée de Marx et le monde social. Mort en 1883, Marx n'a pas connu l'ère des partis de masse - la SPD devenant réellement puissante à partir de la fin des années 1880 - alors même que la prise en compte de cette nouvelle réalité a été une des préoccupations constantes d'Engels, comme le montrent les textes récemment édités par la MEGA et présentés ci-dessus. Si toute projection sur ce que « Marx aurait fait » s'il avait vécu plus longtemps est parfaitement vaine, il parait important de souligner combien Marx lui-même a cherché à synthétiser et vulgariser ses approches les plus complexes dans un objectif directement politique.

La même remarque s'applique à l'histoire de la production intellectuelle. Karl Korsch et Györgi Lukacs ont eu tous les deux une implication importante dans des courants politiques, bien que leurs trajectoires soient fort différentes; de ce point de vue, leur réception, ou plus prosaïquement leur lectorat, est difficilement comparable à d'autres figures majeures comme Theodor W. Adorno. Louis Althusser, membre du PCF, s'il fut toujours à la marge de l'appareil et des processus de décision politique, a été lu par de nombreux militants, dimension éludée par nombre d'études qui lui sont consacrées. À ce sujet, s'il est légitime comme le fait Jan Hoff de critiquer les approches les plus caricaturales de Perry Anderson ne percevant plus rien d'intéressant dans le marxisme d'Europe continentale à partir des années 1980, la grande césure qu'opérait ce dernier quelques années plus tôt ${ }^{33}$ entre les intellectuels marxistes, liés organiquement à une organisation politique de l'entre-deux-guerres et le renouveau théorique des années 1970 émanant d'individus aux rapports bien plus lâches avec les organisations politiques, si elle reste focalisée sur la sphère intellectuelle, mérite d'être réexaminée.

Dans le même esprit, en suivant cette problématique des appropriations de Marx par les organisations politiques et les milieux militants en Allemagne, on pourra être surpris de ne pas trouver de référence aux conséquences de la discussion sur la « liquidation » du marxisme dans la SPD à la suite du congrès de Bad-Godesberg en 1959. Il s'agit pourtant d'un fait historique d'importance, pris désormais pour référence par de nombreux socialistes européens, en particulier en France où il est régulièrement évoqué pour interroger la persistance sur le long terme d'un socialisme français inspiré par le marxisme ${ }^{34}$. En effet, malgré cette hostilité revendiquée depuis 1959, étroitement liée à l'existence de la RDA et du SED, il faut interroger les héritages marxistes qui ne s'expriment pas toujours ouvertement et tenter de comprendre les modalités de la disparition de cette référence, moins évidente et prévue que ne veut le faire croire l'historiographie, souvent portée à relever sans nuance la «décision» de ne plus se 
référer à Marx, sans souligner les débats contradictoires préparatoires et ultérieurs à Bad-Godesberg que permet de saisir une étude attentive des brouillons de textes préalables au célèbre congrès ${ }^{35}$. Certes le statut théorique de ces discussions n'est pas comparable aux débats érudits sur les manuscrits du Capital; mais en tant que mobilisation de la référence à Marx dans l'espace politique, elles s'inscrivent pourtant comme une lecture de Marx à une large échelle. La légitimité d'une telle prise en compte nous semble par ailleurs motivée par des travaux d'histoire sociale portant sur l'importance de l'Arbeiterkultur en Allemagne ${ }^{36}$ : en effet, l'abandon des références marxistes ne doit pas faire oublier que le Parti social-démocrate va continuer en RFA à structurer la classe ouvrière allemande à une échelle de masse pendant au moins deux décennies, dans un contexte de prospérité économique. Bien que modérée politiquement, la social-démocratie repose alors partiellement encore sur une base de classe, telle que celle-ci existe depuis les années 1880-1890, les bouleversements les plus radicaux n'intervenant que dans les années $1980^{37}$; on peut alors légitiment s'interroger sur ce que ce type de structuration "classiste» du monde social doit à l'héritage marxiste, et ce malgré l'abandon explicite de toute référence à la révolution sociale.

21 La même démarche pourrait s'appliquer aux sciences sociales, parfois influencées par le marxisme sans que ce soit explicite la référence à Marx. Ainsi certains courants actuels comme « l'histoire globale » en Allemagne viennent d'une tradition ancienne qui a partie liée avec l'histoire du marxisme, puisqu'un historien majeur de l'ex-RDA comme Walter Markov avait pour référence centrale Marx et Engels et discutait de leur apport pour l'histoire comparée, bien au-delà du seul canon marxiste-léniniste imposé38. Il y a donc des lectures et influences plus «souterraines » de Marx dont l'histoire reste à écrire et qui expliquent partiellement - et ont vraisemblablement facilité - les modalités de l'intérêt actuel pour un des esprits les plus influents de l'histoire mondiale.

\section{«Mille marxismes »?}

22 La publication de ces ouvrages montre un réel intérêt pour Marx, mais qui reste relatif au regard de la période étudiée par Jan Hoff et Ingo Elbe ; il est de plus parfois largement dépendant de facteurs extérieurs ; ainsi l'intérêt grandissant pour les post-colonial studies justifie les travaux sur le Marx «non-occidental». À prendre d'autres problématiques concernant un sujet jadis très étudié dans les deux Allemagnes et en Autriche, à savoir les développements des mouvements ouvriers du temps de Marx et Engels et jusqu'en 1914, les publications sont loin de rencontrer le même écho alors même que, en parallèle de la MEGA, paraissent des sources fondamentales ${ }^{39}$.

Des entreprises pérennes comme la MEGA ou l'Historisch-kritische Wörterbuch des Marxismus assurent dans le milieu intellectuel et académique une visibilité à long terme des travaux sur Marx et les marxistes. Le rapport au champ politique paraît en revanche nettement plus complexe, malgré les faits mentionnés dans notre introduction. Les prétentions à l'interprétation exacte du marxisme ayant par ailleurs disparu, l'expression forgée par André Tosel de " milles marxismes ${ }^{40}$ » s'applique bien également à l'Allemagne, tant les recherches et réflexions à partir de Marx semblent éclatées et avoir renoncé à constituer le cœur d'une définition stricte du monde social et politique. Constat qui rend difficile de percevoir la formation d'un nouveau marxisme ou néo-marxisme cohérent et structuré. Marx est-il désormais pour autant un «classique » que l'on peut facilement 
ranger dans une bibliothèque? On peut légitimement en douter, au regard des deux siècles qui nous séparent de sa naissance.

\section{NOTES}

1. Principale organisation de la gauche radicale allemande issue de la fusion de la PDS (issue du SED est-allemand) et de la WASG (scission de gauche du SPD). Après un bon score aux élections législatives (76 députés en 2009) Die Linke semble en grande difficulté.

2. D'importants articles ont paru dans les Beiträge zur Marx Engels Forschung (Argument) qui permettent de connaître mieux certains de ces épisodes, grâce à l'utilisation des archives des exInstituts du marxisme-léninisme de Berlin et Moscou. De notre côté nous avons apporté quelques éclairages sur la France dans "Éditer Marx en France: mission impossible ?», La Revue internationale des livres et des idées, $\mathrm{n}^{\circ} 16$, mars-avril 2010, p. 52-55.

3. Le tout dernier volume n'était pas parvenu à la date de rédaction de cet article (juin 2012). Pour la liste des volumes voir le site officiel: http://www.bbaw.de/bbaw/Forschung/ Forschungsprojekte/mega/en/Startseite

4. Seule cette section est presque achevée.

5. A noter également que tous les textes étaient publiés directement en allemand, y compris ceux rédigés directement par Marx et Engels dans d'autres langues.

6. La Chine populaire et la Japon disposent tous deux de projets similaires à la MEGA.

7. Marx-Engels Jahrbuch, Berlin : Akademie Verlag, 2003. Moon-Gil Chung, Die deutsche Ideologie und MEGA-Arbeit, Seoul, Moonji Publishing, 2007 (en allemand).

8. 7 octobre 1998 cité par Louis Janover, Marx et les nouveaux phagocytes, Paris, Éditions du Sandre, 2012, p. 230-231.

9. Karl Marx, Friedrich Engels, Werke - Artikel - Entwürfe. März 1883 bis September 1886, Berlin : Akademie Verlag, 2011, 1154 p. Karl Marx, Friedrich Engels, Werke ·Artikel · Entwürfe. März 1891 bis August 1895, Berlin : Akademie Verlag, 2010, 1590 p.

10. cf. Louis Janover, op. cit.

11. Michael Werner et Bénédicte Zimmermann, «Penser l'histoire croisée : entre empirie et réflexivité » in : Annales. Histoire, sciences sociales, 1/2003, p. 7-34.

12. Tristram Hunt, Engels. Le gentleman révolutionnaire, Paris: Flammarion, 2009 (traduit de l'anglais).

13. Marx-Engels Jahrbuch, Berlin : Akademie Verlag, 2009, 238 p. et 2010, 220 p. Le volume de 2011 n'était pas publié à la date de rédaction de cet article.

14. Kevin B. Anderson, Marx at the Margins : On Nationalism, Ethnicity, and Non-Western Societies, Chicago : University of Chicago Press, 2010.

15. En français voir le volume Maurice Godelier (dir.), Sur les sociétés précapitalistes, Paris : Éditions Sociales, 1970.

16. Skadi Krause, « Marx' Russlandbild » in : Marx-Engels Jahrbuch, 2010, p. 53-69.

17. Lucia Pradella, «Kolonialfrage und vorkapitalistische Gesellschaften: Zusätze und Änderungen in der französische Ausgabe des ersten Bandes des Kapital (1872-75) » in : MarxEngels Jahrbuch, 2010, p. 82-100.

18. Claudia Reichel, « Kevin B. Anderson : Marx at the Margins : On Nationalism, Ethnicity, and NonWestern Societies », Marx-Engels Jahrbuch, 2010, p. 189-198. 
19. Karl Marx, Salaires, prix et profit, Paris : Entremonde, 2010, 95 p.

20. Karl Kautsky, Karl Marx' ökonomische Lehren, Stuttgart : Dietz, 1887, 268 p.

21. Rolf Peter Sieferle, Karl Marx zur Einführung, Hamburg: Junius, 2007, 233 p. À noter, une réimpression en 2011.

22. Ibid., p. 192-193.

23. Ibid., p. 198.

24. Ibid., p. 212.

25. Jan Hoff, Marx global. Zur Entwicklung des internationalen Marx-Diskurses seit 1965, Berlin : Akademie Verlag, 2009, 345 p.

26. Ibid., p. 103.

27. Cf. ci-dessus p. XX.

28. Ibid., p. 121.

29. Ibid., p. 138-139.

30. Ingo Elbe, Marx im Westen. Die neue Marx-Lektüre in der Bundesrepublik seit 1965, Berlin: Akademie Verlag, 2010, 643 p.

31. Une telle qualification mériterait discussion dans la mesure où "l'histoire conceptuelle" en Allemagne est souvent considérée en rapport avec l'histoire sociale. Voir notamment les travaux de Reinhart Koselleck.

32. Jan Hoff, « Marx in Germany », Socialism and Democracy, 2010, p. 175-180.

33. Perry Anderson, Sur le marxisme occidental, Paris : Maspero, 1978, 167 p.

34. Cf. notamment les développements dans l'ouvrage Alain Bergounioux et Daniel Cohen (dir.), Le socialisme à l'épreuve du capitalisme, Paris : Fayard/Fondation Jaurès, 2012, 384 p.

35. Karim Fertikh, «Trois petits tours et puis s'en va... Marxisme et programme de Bad Godesberg du Parti social-démocrate allemand ", Sociétés contemporaines, mars 2011, 1, n 81, p. 61-80.

36. Dominique Herbet (ed.), Culture ouvrière - Arbeiterkultur. Mutations d'une réalité complexe en Allemagne du XIXe au XXIe siècle, Villeneuve d'Ascq : Septentrion, 2011, 430 p.

37. op. cit., p. 324.

38. Matthias Middel, Katja Naumann, "L'histoire globale en Allemagne " in : Revue de l'IFHA, 2010, p. 247-284.

39. Voir par exemple les correspondances entre Bernstein et Kautsky : Till Schelz-Brandenburg (ed.), Eduard Bernsteins Briefwechsel mit Karl Kautsky (1895-1905), Frankfurt/Main - New York: Campus Verlag, 2003, 1159 p.

40. André Tosel, « Devenirs du marxisme 1968-2005 : de la fin du marxisme-léninisme aux mille marxismes », en ligne sur www.marxau21.fr (université de Paris I).

\section{AUTEUR}

\section{JEAN-NUMA DUCANGE}

Jean-Numa Ducange est maître de conférences en histoire contemporaine à l'université de Rouen. 